\title{
Increasing the reliability of electrical equipment in the agricultural and industrial complex based on measuring the electrical capacitance of capacitors
}

\author{
Vladimir Vorzhev ${ }^{1}$, Nikolay Rudenko, ${ }^{1, *}$, Pavel Malko', and Vladimir Dergousov ${ }^{1}$ \\ ${ }^{1}$ Don State Technical University, Gagarin Sq., 1, Rostov-on-Don, 344003, Russia
}

\begin{abstract}
The article presents elaborated technical suggestions for increasing reliability of electrical equipment of agro-industrial complexes. The analysis of the already known ways of measuring electric capacitance is carried out and it demonstrates the necessity of withdrawing a capacitor from a working circuit for sufficient accuracy of determining electric capacitance. The urgency of developing a method for measuring electric capacitance without removing the capacitor from a working circuit to reduce the average recovery time is shown. Theoretical foundation of the possibility to measure electric capacitance without a capacitor being withdrawn from a working circuit in smoothing filter mode is worked out; a structural electric circuit of an electric capacitance measuring device is elaborated and the principle of its operation is described; methods of measurement by means of the given device are elaborated; conditions for creating and maintaining the measurement mode of the device are substantiated. The validity of the received results is checked with the help of the programmed package Mathcad, as well as on electric models of the main blocks of the device. The obtained results can be used to measure electric capacitance of electrolytic capacitors during maintenance and electrical equipment renovation and also can promote its reliability.
\end{abstract}

\section{Introduction}

Efficiency of an agro-industrial complex (AIC) can be improved by automation and electrification of all technological processes. In particular, power installations and various electro-technical equipment containing capacitors are widely used in AIC. They include starting controllers of electric drives, heating and lighting systems, smoothing filters of power converters and power units, reactive power compensation devices, power factor correction devices and many others [1,2].

However, capacity of electrical capacitors decreases due to their natural wear, which leads to poorer performance of power installations.

Thus, electrolytic capacitors eventually deteriorate because electrolytes dry out. This mainly concerns aluminum capacitors, however, small-capacity tantalum capacitors are

* Corresponding author: rnv.2017@mail.ru 
prone to wear as well $[3,4]$. Nonetheless, they appear to have no visible flaws, which makes diagnostics significantly more complicated. Therefore, to control electrical capacity of condensers is a critical maintenance and renovation operation $[5,6]$.

One of the possible ways to increase the reliability of electrical equipment of AIC is improving availability rate at the expense of reduced average recovery time [7-10]. However, the analysis of the already known methods of measuring electrical capacitance shows that to reach sufficient measurement accuracy requires withdrawal of a capacitor from a measuring RC-circuit [11-16]. This leads to an increase in the average recovery time. Therefore, to resolve the existing contradiction, it is urgent to develop a method for measuring electrical capacitance, which makes it possible to reduce the average recovery time.

\section{Research material}

The objective of the given article is elaboration of technical suggestions for improving reliability of electric equipment of AIC on the basis of measuring electrical capacitance without withdrawing a capacitor from a working circuit. To attain this objective, it seems reasonable to complete the following particular tasks:

- To provide theoretical foundation of the possibility to measure electrical capacitance of a capacitor without withdrawing it from a working circuit in smoothing filter mode,

- To elaborate a structural circuit of an electrical capacity measurement device and describe the principle of its work,

- To work out a method of measuring electrical capacitance without withdrawal of a capacitor from a working circuit in smoothing filter mode,

- To provide justification of serviceability conditions and accuracy of measurement which is sufficient for solving engineering tasks by means of the given device.

\subsection{Theoretical foundation}

Theoretical foundation of the possibility to measure electrical capacitance without withdrawing a capacitor from a working circuit in smoothing filter mode. It is known that while a capacitor is working on quite a high frequency (for which the ratio $X_{C}<<R$ is met), voltage on its plates $u_{C}(t)$ represents a certain superposition of the dc component $U_{0}$ and the ac component, the amplitude of which is, as a rule, much lower than the quantity $U_{0}$, which is $U_{m}$ pulse $<<U_{0}$. This electric condition of a capacitor is in keeping with smoothing filter mode, the circuit of which is shown on Fig. 1.

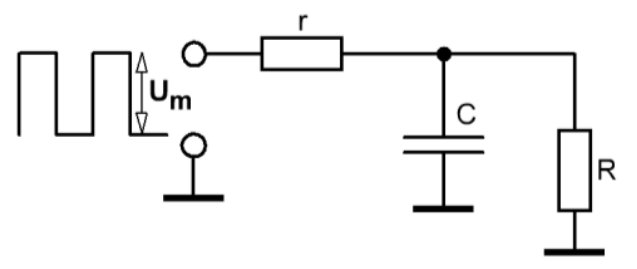

Fig. 1. A typical circuit of engaging a capacitor into a smoothing filter circuit.

Apart from that, if transient stationary processes are established and under certain conditions the shape of the ac component is known to be triangle (i.e. linear), as is shown on Fig. 2. This signal waveform evidently means that upon charge and discharge exponential time dependences can be to a first approximation replaced with linear ones with insignificant sacrifice of ac-curacy of description of the given process. 


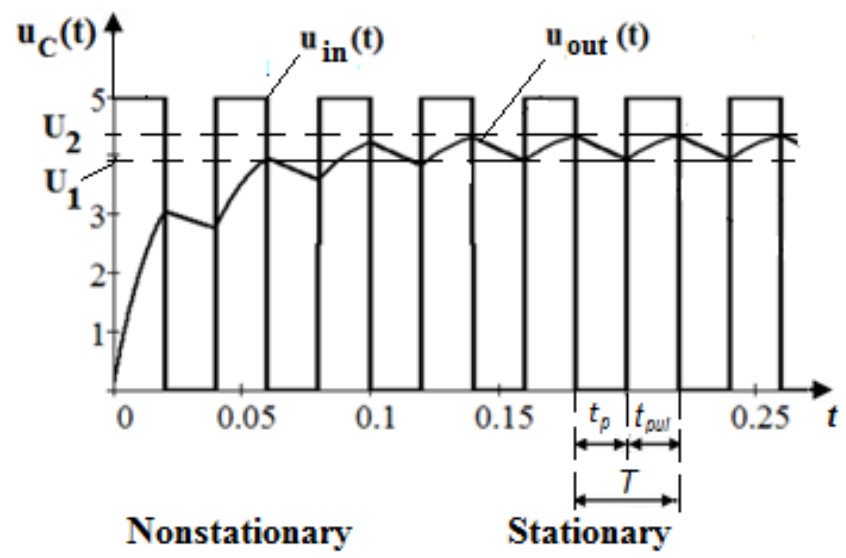

Fig. 2. Dynamics of voltage changes on capacitor plates during operation in smoothing filter mode under exposure to a source of rectangular pulses.

By means of the theory of describing transient processes in linear electrical circuits we obtain an analytical expression for the value of the ripple factor of the smoothing filter shown on Figure 1 under the condition of preserved linear shape of the ac voltage component $u_{C}(t)$.

It is known that the process of charge of such a circuit being under action of rectangular pulses is described via the following expression (1):

$$
u_{\text {Char }}=\frac{R \cdot U_{m}}{r+R}\left(1-e^{-\frac{t}{\tau_{\text {char }}}}\right) ; \quad \tau_{\text {char }}=\frac{r \cdot R \cdot C}{r+R},
$$

where $\tau_{c h a r}$ - relaxation time of a $R C$-circuit in the charging procedure.

Discharge procedure of this circuit is described via expression (2):

$$
u_{\text {Cdis }}=\frac{R \cdot U_{m}}{r+R} e^{-\frac{t}{\tau_{d i s}}} ; \quad \tau_{d i s}=R \cdot C,
$$

where $\tau_{\text {dis }}$ - relaxation time of a $R C$-circuit in the discharging procedure.

Expression (1) is now modified on the assumption of a stationary transient process going on where periodic voltage modulation $u_{C}(t)$ occurs within voltage intervals from $U_{1}$ to $U_{2}$ (Fig. 2).

Full solution of a charging process differential equation looks as follows:

$$
u_{\text {Char }}(t)=A e^{-\frac{t}{\tau_{\text {char }}}}+\frac{R \cdot U_{m}}{r+R} .
$$

In order to determine the unknown constant $A$ we consider voltage to be equal to $U_{1}$ at $t$ $=+0$ according to commutation laws. Therefore, according to (3):

$$
A=U_{1}-\frac{R \cdot U_{m}}{r+R} .
$$

By completing (3) with (4), we obtain: 


$$
u_{\text {Cchar }}(t)=\left(U_{1}-\frac{R \cdot U_{m}}{r+R}\right) e^{-\frac{t}{\tau_{c h a r}}}+\frac{R \cdot U_{m}}{r+R} .
$$

Then, on impulse time expiry $t_{p u l}$, the capacitor will charge up to voltage $U_{2}$, which is:

$$
U_{2}=\left(U_{1}-\frac{R \cdot U_{m}}{r+R}\right) e^{-\frac{t_{p u l}}{\tau_{c h i r}}}+\frac{R \cdot U_{m}}{r+R} .
$$

Next, as impulse expires and a pause with duration $t_{p}$ starts, voltage on the capacitor will drop to $U_{I}$ again, which is:

$$
U_{1}=U_{2} e^{-\frac{t_{p}}{\tau_{\text {dis }}}} .
$$

Expressions (6) and (7) represent the equation system regarding the unknown $U_{I}$ and $U_{2}$, the solution of which is shown below.

$$
\begin{gathered}
U_{1}=\frac{R \cdot U_{m}}{r+R} \cdot \frac{e^{-\frac{t_{p}}{\tau_{d i s}}}-e^{-\frac{t_{p u l}}{\tau_{\text {char }}}} \cdot e^{-\frac{t_{p}}{\tau_{d i s}}}}{1-e^{-\frac{t_{p u l}}{\tau_{c h a r}}} \cdot e^{-\frac{t_{p}}{\tau_{d i s}}}} ; \\
U_{2}=\frac{R \cdot U_{m}}{r+R} \cdot \frac{1-e^{-\frac{t_{p u l}}{\tau_{c h a r}}}}{1-e^{-\frac{t_{p u l}}{\tau_{\text {char }}}} \cdot e^{-\frac{t_{p}}{\tau_{d i s}}}} .
\end{gathered}
$$

We suggest that due the conditions of transient processes the voltage shape on the capacitor be considered linear. Then exponential character of time dependences can be represented as linear by means of a Taylor series expansion up to first-order summands:

$$
e^{-a x} \approx 1-a x, \quad x \rightarrow 0 .
$$

Then, the duty cycle of the rectangular pulses source is supposed to equal to 2 , i.e. $t_{p u l}=$ $t_{p}=T / 2$.

In this case expressions (8) and (9) become:

$$
\begin{gathered}
U_{1}=\frac{R \cdot U_{m}}{r+R} \cdot \frac{\tau_{d i s}}{\tau_{c h a r}+\tau_{d i s}-T / 2} \cdot\left(1-\frac{T / 2}{\tau_{d i s}}\right) ; \\
U_{2}=\frac{R \cdot U_{m}}{r+R} \cdot \frac{\tau_{d i s}}{\tau_{c h a r}+\tau_{d i s}-T / 2} .
\end{gathered}
$$

By means of expressions (11) and (12), and allowing for the linear shape of the signal on capacitor clamps the constant component value of signal $U_{0}$ and pulse amplitude $U_{\mathrm{m}}$ pulse is determined:

$$
U_{0}=\frac{U_{1}+U_{2}}{2}=\frac{R \cdot U_{m}}{r+R} \cdot \frac{\tau_{d i s}-T / 4}{\tau_{c h a r}+\tau_{d i s}-T / 2}
$$




$$
U_{\text {mpul }}=\frac{U_{2}-U_{1}}{2}=\frac{R \cdot U_{m}}{r+R} \cdot \frac{T / 4}{\tau_{\text {char }}+\tau_{\text {dis }}-T / 2} .
$$

Then the pulse coefficient of this signal is:

$$
K_{p}=\frac{U_{m p u l}}{U_{0}}=\frac{T / 4}{\tau_{\text {dis }}-T / 4} .
$$

In the strict sense, expression (15) is not a pulse coefficient because it is the fundamental harmonic amplitude $U_{m}$ main that must be in the numerator of this expression. However, it is not critical from the point of view of determining the electrical capacitance value, since practical value of expression (15) lies in its being determined only by the discharge time of the circuit under study $\tau_{d i s}=R C$, which makes it possible (with known values of resistance $R$ and pulse repetition frequency $f_{p u l}$ ) to determine the electrical capacitance value $C$ with sufficient accuracy for engineering calculations.

As it can be seen from (15) it is more convenient to use value $1 / K_{p}$ for programming a microcontroller, as its use leads to a simpler expression for value determination:

$$
\frac{1}{K_{P}}=\frac{4 \tau_{\text {dis }}}{T}-1 .
$$

In this case, the required value of electric capacitance $C$ will be determined via the following formula:

$$
C=\frac{T}{4 R} \cdot\left(\frac{1}{K_{p}}+1\right)=\frac{1}{4 R \cdot f_{p u l}} \cdot\left(\frac{1}{K_{p}}+1\right),
$$

where $f_{p u l}$ is rectangular pulse repetition frequency of a duty cycle equal to 2 .

Therefore, given the values of resistance $R$ and pulse repetition frequency $f_{\text {pul }}$, and also the known (measured) values of the signal $U_{0}$ dc component and pulse amplitude $U_{\mathrm{m}}$ pulse, electric capacitance can be calculated according to formulas (15) and (17).

\subsection{Elaboration of a structural electric circuit of the electric capacitance measuring device and description of its operation principle}

As it was mentioned above, the work principle of the device is to measure the following quantities: the dc component $U_{0}$ and voltage pulse amplitude $U_{\mathrm{m}}$ pulse on capacitor clamps with the known value of $k^{\text {th }}$ rectangular pulse repetition frequency $f_{p u l} k$. At the same time the necessary signal form is achieved by monitoring quantity $U_{m}$ pulse, the value of which must always be lower than a certain limit quantity $U_{m} \max$, determined by means of numerical calculations for chosen measuring sub-bands.

The electric structural circuit of the device is shown on Fig. 3. Forming of rectangular pulse repetition with fixed frequency $f_{\text {pul }}$ (there are 4 clock frequencies in the given circuit) is carried out by means of a clock frequency generator (CFG), the output of which is connected to a clock input of a frequency divider (FD), which initiates count pulses on its outputs $Q 1, Q 2, Q 3$ corresponding to frequencies $f_{p u l} / 2, f_{p u l} / 4, f_{p u l} / 8$, respectively. These signals arrive to multiplex inputs (M) $I 1, I 2, I 3$ (a signal with frequency $f_{p u l}$ arrives to input I0 directly from the CFG output), and pulses from certain inputs with a number 
corresponding to a control word preset by driving points $A 0, A 1$ appear on the multiplex output (Y).

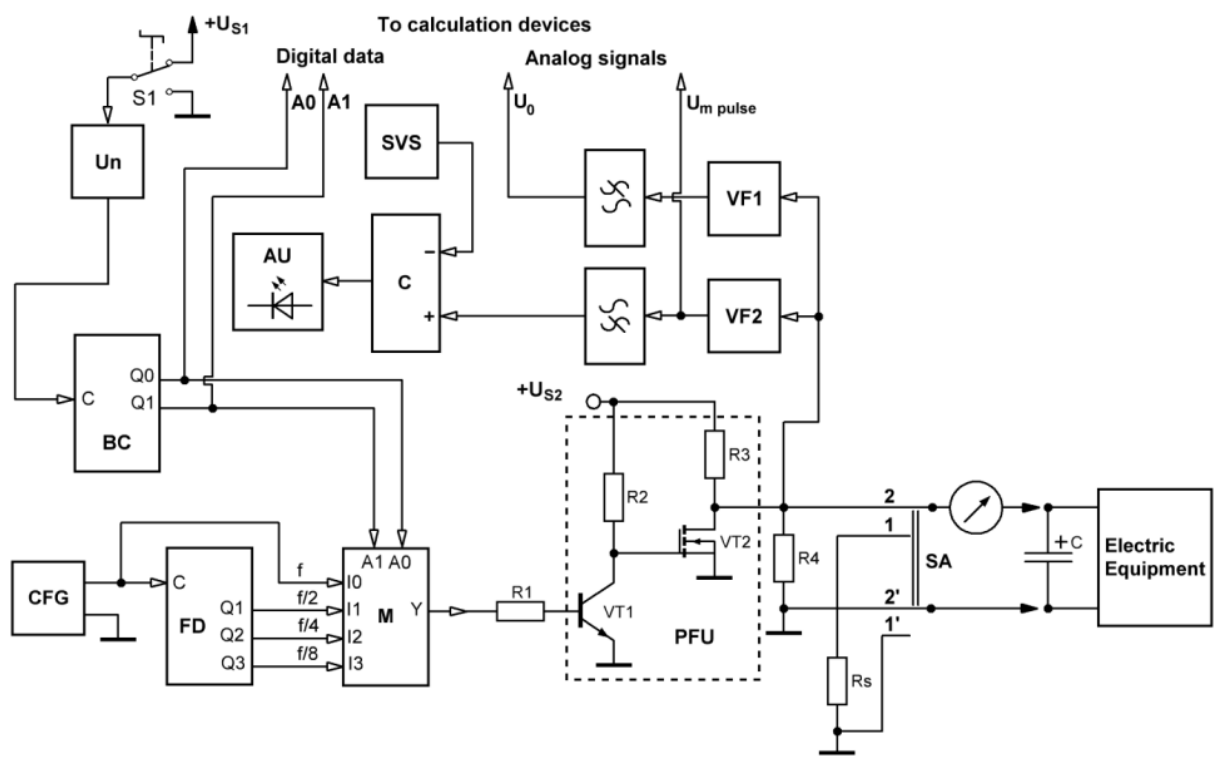

Fig. 3. A structural circuit of a measuring device of electric capacitance of a capacitor.

The choice of frequency $f_{\text {pul } k}$ is carried out by means of a univibrator (Un), which generates single rectangular pulses of fixed width coming from its output to the input of a binary counter $(\mathrm{BC})$ and making the figure on its output increase by 1 . Counting in a $\mathrm{BC}$ can only reach a number corresponding to the number of possible frequencies, then it clears the registers.

Rectangular pulse sequence of the chosen frequency $f_{\text {pul } k}$ comes to a pulse forming unit (PSU) consisting of a two-stage transistor switch (VT1,VT2) and a measuring chain of a $R C$-circuit $(R 3, R 4, C)$, where $\mathrm{C}-$ is a capacitor of required capacitance.

Then, through voltage followers $V F 1, V F 2$ (assembled on operational amplifier microcircuits can serve this function as well) the measuring signal comes to the forming units of the dc and ac components.

Forming of the dc component occurs by means of a low-pass filter intended for a minimal pulse repetition frequency $f_{\text {pul min }}$. Forming of the ac component occurs with the help of a high-pass filter, also intended for a minimal pulse repetition frequency $f_{\text {pul min }}$.

Monitoring the value of pulse amplitude is carried out via a comparator (C) which at any one time compares half-period average voltage (and amplified up to the level where voltage drop on a rectifier diode does not make a significant impact on its value) of the ac component $U_{\text {aver }}$ with certain standard voltage $U_{s t}$, removed from the output of the reference voltage source (RVS). While the condition $U_{\text {aver }}<U_{s t}$ is fulfilled, voltage on the comparator output is at a low level and an alarm unit (AU) connected to its output does not give a light or (and) sound signal. But if the condition $U_{\text {aver }}>U_{s t}$ is fulfilled, a high-level signal appears on the comparator output, which supplies the alarm unit with electricity. It shows to the operator that this frequency is inapplicable for a RC-circuit in measuring mode.

After that, by short-term pressing of key S1 the operator gives a single pulse to the input of the binary counter $(\mathrm{BC})$, therefore increasing the control word on the multiplex driving points $(A 0, A 1)$ by 1 . Then the whole process repeats until the condition $U_{\text {aver }}<U_{s t}$ is fulfilled and the AU does not give a light and (or) a sound signal. It shows to the operator 
that the chosen frequency corresponds to the measured capacitance and that the device turns to measurement mode.

In electric capacitance measuring mode the input of a calculating device (CD) (not shown in the figure) receives a digital signal corresponding to the chosen frequency $f_{\text {pul } k}$, as well as analog signals corresponding to the dc component of signal $U_{0}$ and pulse amplitude $U_{\mathrm{m} \text { pul. }}$ A CD can be assembled on the basis of a microcontroller with analog-digital transformers and a memory device which contains an electric capacitance calculation programmed according to formulas (15), (17) and the value of resistance $R 4$. The calculation result can be brought onto the input of a digital indication for the operator to take the indications.

\subsection{Elaboration of electric capacitance measuring method without withdrawing a capacitor from a working circuit in smoothing filter mode}

The given method contains the following sequence of actions:

- To cut off the electric equipment (EE) (Fig. 3), containing a capacitor with required capacitance from an external electric energy source.

- To connect the device to clamps of a capacitor of required capacitance with the help of probes and remove residual charge by means of switching the SA switch to position 2-2'. At the same time the operator controls discharge current drop of the capacitor to zero with the help of galvanometer P1.

- To switch the SA switch to position 1-1' and choose pulse repetition frequency with the help of the alarm unit AU and they key S1 as described in 2.2.

- To measure the chosen frequency $f_{p u l} k$, the dc component of signal U0, pulse amplitude $U_{m}$ pul, to calculate and take the readings of electric capacitance value.

\subsection{Justification of conditions of efficiency and sufficient accuracy of the given device}

Concerning the conditions that provide voltage of linear shape on capacitor clams, according to (10), they can be expressed by the following ratios:

$$
\frac{T}{2} \square \tau_{\text {char }} ; \quad \frac{T}{2} \square \tau_{d i s} .
$$

However, this condition cannot be fulfilled on one fixed pulse repetition frequency $f_{p u l}$ at determining values of capacitances in a sufficiently broad band. One of the ways to fulfill this condition is to limit pulse amplitude of output voltage with a possibility to select pulse repetition frequency from a prefixed set of $n$ frequencies $f_{\text {pull }}, f_{\text {pul }}, \ldots f_{\text {pul } n}$.

Due to complexity of mathematical transformations the research data were carried out via the application programmed Mathcad, which enabled determining maximum permissible value of pulse amplitude $U_{m} \max$. In its turn, it provides linear shape of the output signal for the fixed band of measuring capacitances $C_{\min } \ldots C_{\max }$. For the given device it is $0,15 \mathrm{~B}$.

Table 1 provides data for measuring sub-bands of capacitances and corresponding clock frequencies obtained by means of calculations in Mathcad. 
Table 1. Measuring capacitance sub-bands and corresponding clock frequencies.

\begin{tabular}{|c|c|}
\hline Measuring sub-bands, $\boldsymbol{\mu F}$ & Clock frequency, $\mathbf{H z}$ \\
\hline $2000-5100$ & 50 \\
\hline $1000-2000$ & 100 \\
\hline $500-1000$ & 200 \\
\hline $250-500$ & 400 \\
\hline
\end{tabular}

\section{Conclusions}

- The possibility of measuring electric capacity without withdrawing a capacitor from a working circuit in smoothing filter mode is proved. Also, calculation ratios are obtained which enable measuring electric capacitance of an electrolytic capacitor by means of altering the dc component and the amplitude of the ac component on its plates, taking into account the linear shape of the ac voltage component.

- A structural circuit of an electric capacitance measuring device without withdrawing a capacitor from a working circuit in the smoothing filter mode.

- A method of measuring electric capacitance without withdrawing a capacitor from a working circuit in smoothing filter mode is elaborated.

- The conditions of efficiency and accuracy sufficient for engineering calculations of the given device, as well as measuring sub-bands of capacitance and their corresponding clock frequencies are justified.

- Introducing of the given technical suggestions on measuring electric capacitance of capacitors without withdrawing a capacitor from a working circuit will promote reliability of electric equipment of agro-industrial complexes.

\section{References}

1. E.A. Kireeva, A full guide on electric equipment and electrical engineering (with calculation examples) (KNORUS, M., 2012)

2. A.I. Potapov, A.A. Pugachev, I.A. Potapov, Control, diagnostics and maintenance of quality of electric energy. A scientific and methodological reference book. 1. Providing quality of electric energy (Publishing house DEAN, SPb, 2014)

3. Shuai Zhao, Shaovei Chen, Huai Wang, Microelectronics Reliability 100-101, 113401 (2019) doi.org/10.1016/j.microrel.2019.113401

4. Gang Joon Kim, Nam Hyun Lee, Jongkyun Kim, Jong Eun Suk, Yunsun Lee, $\begin{array}{llll}\text { Microelectronics } & \text { Reliability } & \mathbf{8 8 - 9 0}, & 179-182\end{array}$ doi.org/10.1016/j.microrel.2018.06.117

5. O.G. Zacharov, Flaw search in contact-relay circuits (Infra-Ingeneria, M., 2017)

6. A.I. Yashchura, Technical maintenance and energy equipment renovation system: a book of reference (ENAS, M., 2013)

7. J. Romero, M.H. Azarian, M. Pecht, Microelectronics Reliability 112, 113725 (2020) doi.org/10.1016/j.microrel.2020.113725

8. M.A. Maher, M. Al-Maghalseh, Procedia Computer Science 130, 744-752 (2018) doi:10.1016/j.proeng.2018.04.129

9. N. Delmonte et al., Microelectronics Reliability 114, 113737 (2020) doi.org/10.1016/j.microrel.2020.113737 
10. Wu Xiaojuan, Wang Yajun, Zeng Qingxuan, Procedia Engineering 45, 998 - 1003 (2012) https:// www.sciencedirect.com

11. Sensors: a book of reference (Technosfera, M., 2012)

12. I.G. Minaev, V.V. Samoilenko, D.G. Ushkur, Method of measuring electric capacity, No. 2660283C1 Russian Federation. No. 2017100189 (2019)

13. Kuo-Liang Teng, Li-Wei Chen, Method for measuring electric capacity of cell module, United States Patent Application 20160146894A1. Application Number: 14/751091 (2016)

14. I.V. Korotkova, O.M. Pavlov, G.P. Telitchenko, V.I. Shevtsov, Method for electric capacity measuring and device for its realisation, No. 2577803 C1 Russian Federation. No. 2014135363/28A (2016)

15. R. Huq, S. Anwar, 19th IFAC World Congress Cape Town, South Africa, 8463-8468 (2014) doi: 978-3-902823-62-5 / 2014.

16. L.E. Helseth, Journal of Energy Storage 35, 102304 (2021) https:// www.elsevier.com/locate/est 